12 Fitzpatrick MC, Floyd K. A systematic review of the cost and cost effectiveness of treatment for multidrugresistant tuberculosis. PharmacoEconomics 2012; 30: 63-80.

13 Esposito S, D’Ambrosio L, Tadolini M, et al. ERS/WHO Tuberculosis Consilium assistance with extensively drug-resistant tuberculosis management in a child: case study of compassionate delamanid use. Eur Respir J 2014; 44: 811-815.

14 Tadolini M, Dolma Lingtsang R, Tiberi S, et al. First case of extensively drug-resistant tuberculosis treated with both delamanid and bedaquiline. Eur Respir J 2016 [in press DOI: 10.1183/13993003.00637-2016].

15 Sandgren A, Cuevas LE, Dara M, et al. Childhood tuberculosis: progress requires an advocacy strategy now. Eur Respir J 2012; 40: 294-297.

\title{
Efficacy and tolerability of ethionamide versus prothionamide: a systematic review
}

To the Editor:

To treat multidrug-resistant tuberculosis (MDR-TB), the World Health Organization recommends to include, during the intensive phase of treatment, at least a parenteral agent, a later-generation fluoroquinolone, ethionamide (Eth) (or prothionamide (Pth)), cycloserine (Cs) or p-aminosalicylic acid (PAS) if Cs cannot be used, and pyrazinamide (Pzd) (which is not considered among the aforementioned four probably effective drugs) $[1,2]$. In particular, among the four drugs likely to be effective, at least two essential or "core" drugs (one with a good bactericidal and one with a good sterilising activity) and two other "companion" drugs should be administered [3,4].

In most of the countries where drug susceptibility testing cannot be performed to guide treatment regimen design, standardised second-line treatment regimens are prescribed, based on kanamycin $(\mathrm{Km})$, levofloxacin (Lfx), Eth, Cs and Pzd. Although the regimen is built following the international recommendations, the outcomes remain poor globally $[5,6]$. Less than $50-70 \%$ of the cases, in fact, achieve treatment success $[5,6]$, resulting in insufficient control of MDR-TB. It is widely recognised that one frequent cause of poor outcome is treatment default, which is mostly due to the low tolerability of the antituberculosis drugs employed [7]. One of the less tolerated antibiotics is Eth, because of the serious and frequent gastric adverse events [8 9] or of hypothyroidism, which is frequently subclinical.

Eth and Pth are thionamide drugs, characterised by a structure similar to isoniazid (Inh). They inhibit the mycobacterial synthesis of mycolic acid through a specific action against the inhA product enoyl-acyl carrier protein reductase; thus, they can be classified as bactericidal. However, their metabolic process is poorly known and, therefore, it is difficult to understand the pathogenesis behind the occurrence of adverse events following their administration.

If the reasons behind the choice of Km, Lfx, Cs and Pzd are in general easy to explain (although they do not necessary represent the best choice), less clear is the reason why Eth is used in the majority of the programmes instead of Pth. For this reason, we have carried out a systematic review on the existing evidence focused on efficacy and tolerability of Eth versus Pth.

The search was performed using the search engine PubMed, without any time restriction. Only articles written in English, French, Spanish and Italian were selected. The following keywords were used to retrieve the scientific references related to the research question: "ethionamide", "prothionamide", "efficacy", "safety" and "tolerability".

Reviews, case reports, case series and letters were excluded. References of the selected articles were analysed in order to identify significant manuscripts not found by the search engine.

We adopted a simplified five-point checklist adapted from that of the Scottish Intercollegiate Guidelines Network to grade the quality of the scientific evidence. A PRISMA (Preferred Reporting Items for Systematic Reviews and Meta-Analysis) flowchart was used to summarise the search and selection process (figure 1).

We found only six articles addressing this issue, all of them published before 1970. The core information derived from these articles is summarised in table 1. In the "double blind" trial by Chambatтe et al. [10], published in 1965 , Pth was compared to Eth $\left(1 \mathrm{~g} \cdot\right.$ day $\left.^{-1}\right)$. Tolerability was reported to be very good by $62 \%$ 

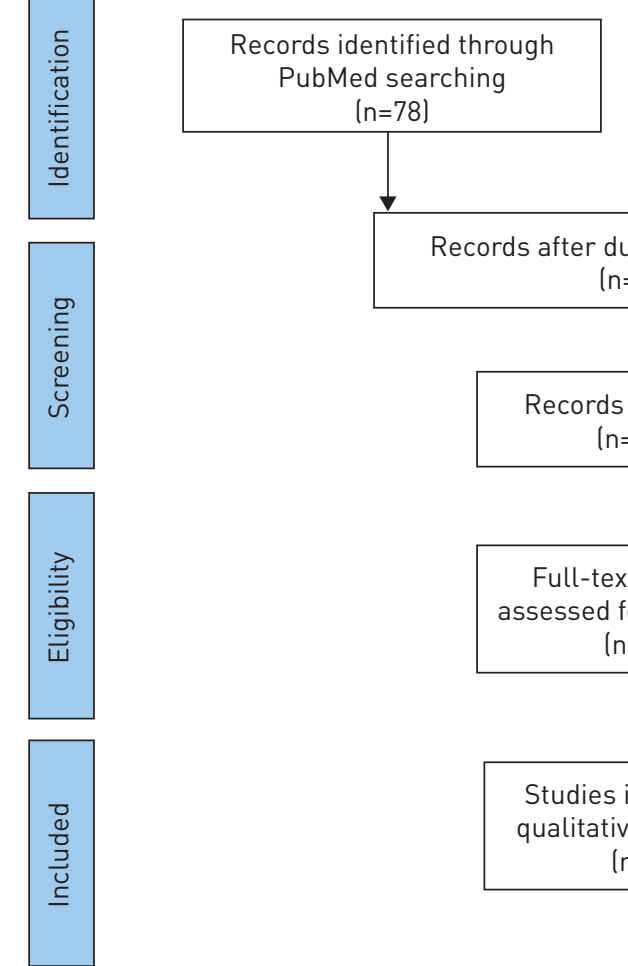

Additional records identified through other sources $(n=4)$

FIGURE 1 Preferred Reporting Items for Systematic Reviews and Meta-Analysis (PRISMA) flow diagram.

patients receiving Pth, while only $24 \%$ of those receiving Eth reported that it was tolerable. Eth and Pth were both prescribed in combination with another two or three antituberculosis drugs, and the regimens were not standardised.

In a Japanese study published in 1968 [11], 531 tuberculosis cases were divided into three groups: in Group 1, patients received streptomycin (Sm), Inh and PAS; in Group 2, Sm, Inh and Eth; and in Group 3, Sm, Inh and Pth. The thionamides were prescribed at a daily dosage of $500 \mathrm{mg}$. The sputum conversion rates were similar in the three groups $(96 \%, 98 \%$ and $96 \%$, respectively), while the rate of adverse events was statistically higher in the Eth arm (75\% versus $60 \%$, respectively). However, no significant difference was reported in terms of treatment interruption. Notably, Group 1 patients, who were not treated with thionamides, showed a toxicity rate of only $32 \%$.

In the double-blind British Tuberculosis Association study, published in 1968 [12], 53 patients receiving Pth $(750 \mathrm{mg})$, in addition to $\mathrm{Sm}$ and $\mathrm{Inh}$, were compared with 48 patients receiving Eth (750 $\mathrm{mg}$ ) and the same backbone drugs for 10 weeks. Gastric intolerance was more frequent in the Eth group (50\%) than in the Pth group (32\%), although the difference was not statistically significant. Additionally, minor adverse events were more frequent with Pth, while severe side-effects were more frequent with Eth, even if the differences were not statistically significant. The liver impairment rate was similar in the two groups. Furthermore, higher weight gain, probably attributed to a better gastrointestinal tolerability of Pth, was found in those who received Pth compared to those receiving Eth.

The double-blinded study by Fox et al. [13], published in 1969, compared the tolerability of Eth and Pth in 128 African patients, using different dosages in intermittent regimens; in addition, the effect of the vitamin B complex in reducing potential adverse events was also evaluated. The incidence and severity of adverse events for Eth (at doses ranging from 0.25 to $1.75 \mathrm{~g}$ daily) and Pth (at doses ranging from 1.25 to $1.75 \mathrm{~g}$ daily) were compared with a placebo-administered group. Females reported more adverse events following exposure to both drugs than males, but the differences between Eth and Pth were not significant. However, males showed significantly $(\mathrm{p}<0.005)$ more adverse events with Eth $(36 \%)$ than Pth $(17 \%)$, with significant differences for gastric intolerance $(p<0.01)$, vomiting $(p<0.01)$ and headache $(p<0.003)$. Furthermore, one or more adverse events occurred more frequently when the dosage of Eth increased, but a trend between dosage and side-effects was not observed in the case of Pth. The addition of the vitamin B complex had no effect on the incidence of new adverse events. 
TABLE 1 Findings and characteristics of the available studies comparing tolerability and efficacy of ethionamide (Eth) versus prothionamide (Pth)

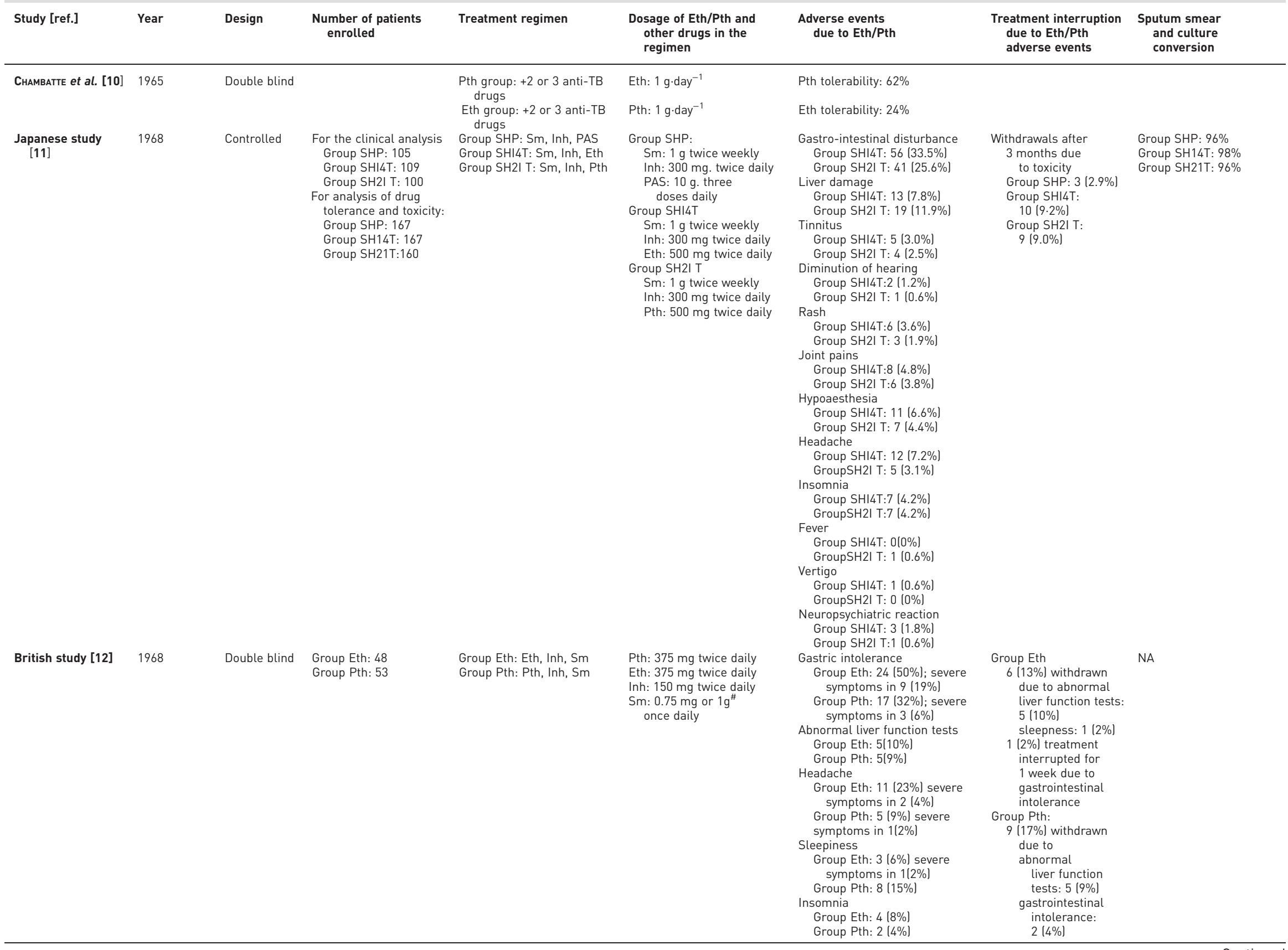




\begin{tabular}{|c|c|c|c|c|c|c|c|c|}
\hline Study [ref.] & Year & Design & $\begin{array}{l}\text { Number of patients } \\
\text { enrolled }\end{array}$ & Treatment regimen & $\begin{array}{l}\text { Dosage of Eth/Pth and } \\
\text { other drugs in the } \\
\text { regimen }\end{array}$ & $\begin{array}{l}\text { Adverse events } \\
\text { due to Eth/Pth }\end{array}$ & $\begin{array}{l}\text { Treatment interruption } \\
\text { due to Eth/Pth } \\
\text { adverse events }\end{array}$ & $\begin{array}{c}\text { Sputum smear } \\
\text { and culture } \\
\text { conversion }\end{array}$ \\
\hline Fox et al. [13] & 1969 & Double blind & $\begin{array}{l}\text { Group A-B: } 6 \text { ( } 49 \text { days) } \\
\text { Group C-D: } 6 \text { (49 days) } \\
\text { Group E-H: } 6 \text { ( } 49 \text { days) } \\
\text { Group I-L: } 20 \text { ( } 21 \text { days) }\end{array}$ & $\begin{array}{l}\text { Group A-B: Sm, Inh, Eth, ST } \\
\text { Group C-D: Sm, Inh, } \\
\text { Eth, ST } \\
\text { Group E-H: Sm, Inh, Eth, } \\
\text { Pth, ST } \\
\text { Group I-L: Sm, Inh, Eth, Pth, } \\
\text { vitamin B complex additive }\end{array}$ & 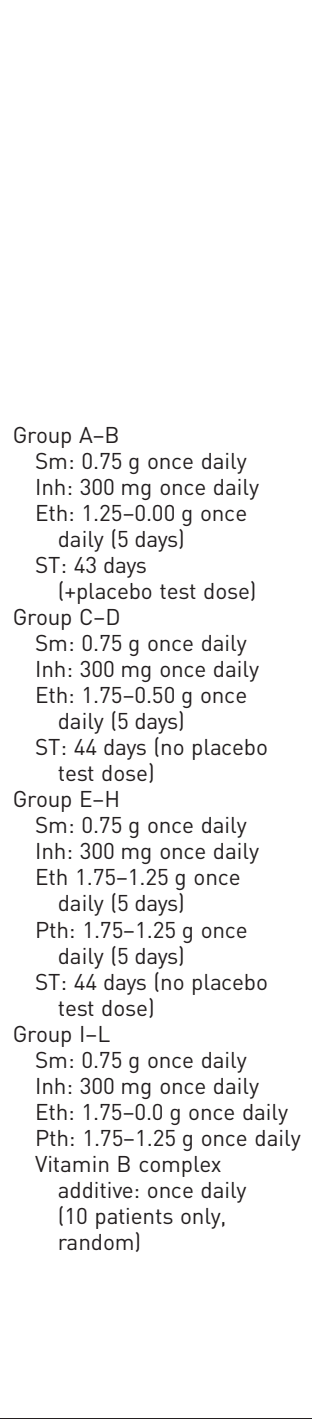 & 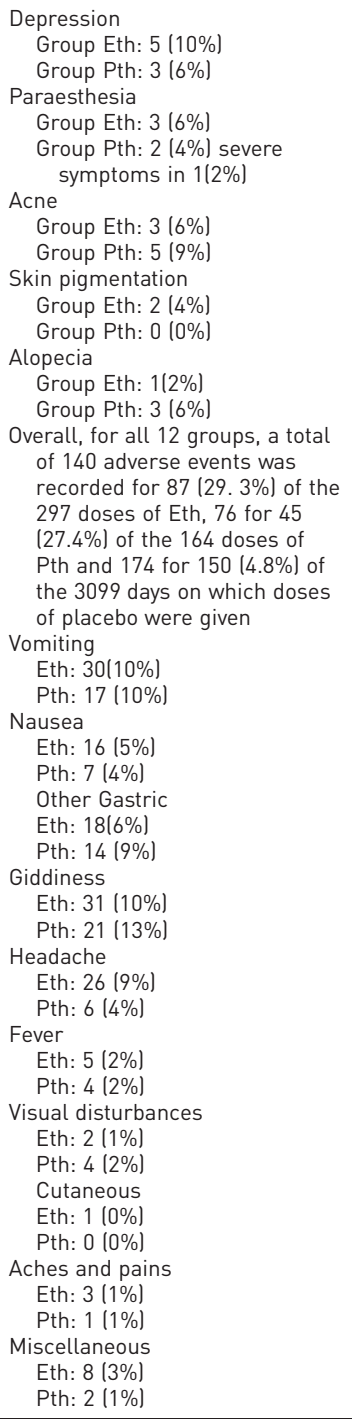 & $\begin{array}{l}\text { acute psychosis: } 1 \\
\text { paraesthesia: } 1(2 \%) \\
1(2 \%) \text { treatment } \\
\text { interrupted for } 1 \text { week } \\
\text { due to severe } \\
\text { headache }\end{array}$ & No data \\
\hline
\end{tabular}




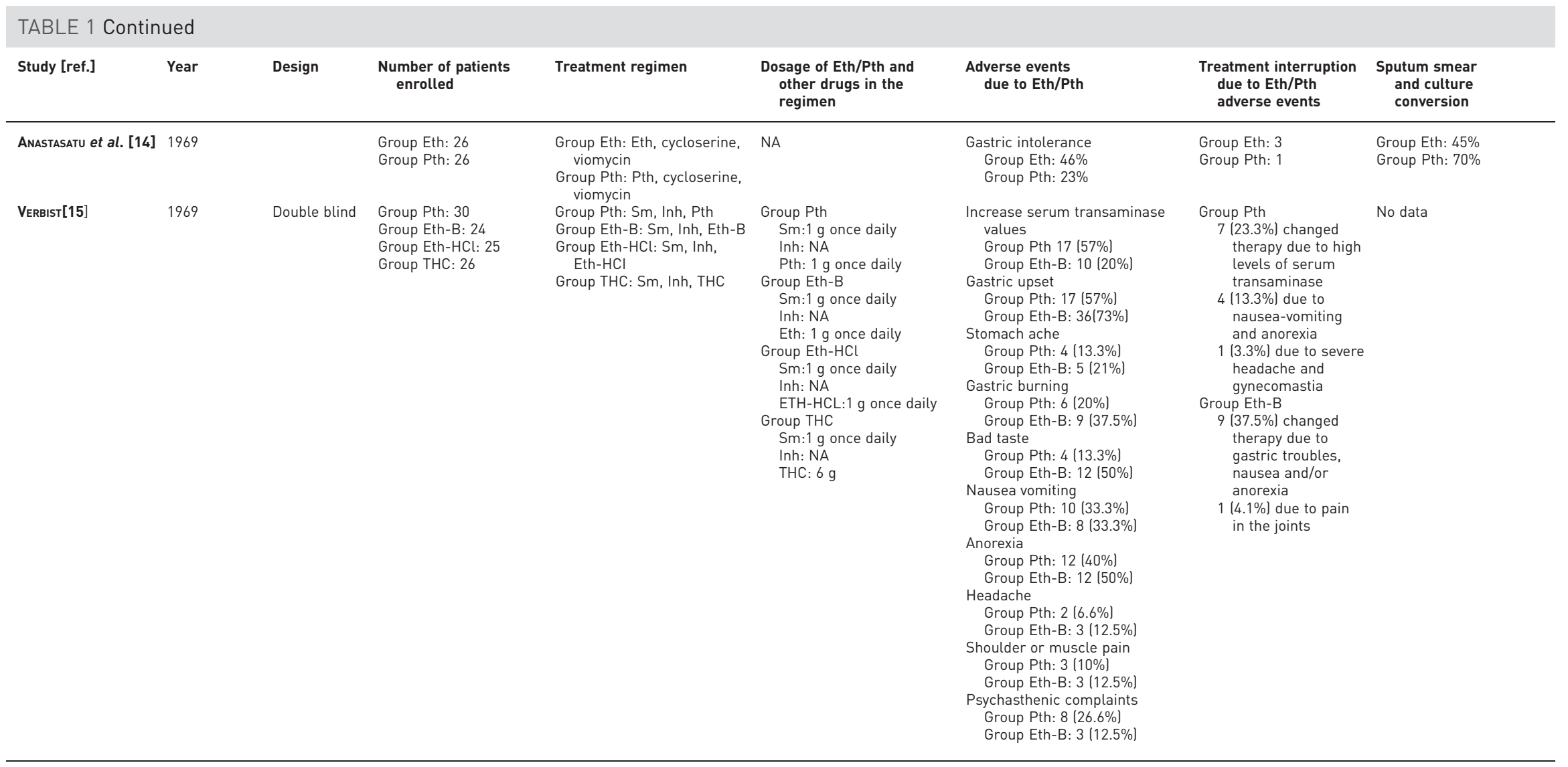

TB: tuberculosis; SHP: streptomycin, isoniazid and sodium p-aminosalicylate; SHI4T: streptomycin, isoniazid and Eth; SH2I T: streptomycin, isoniazid and Pth; Sm: streptomycin; Inh: isoniazid; PAS: p-aminosalicylic acid; NA: not available; ST: supplement tablets (placebo); Eth-B: ethionamide base; Eth-HCl: ethionamide hydrochloride; THC: thiocarlide (control).

\#: dose of Sm was left to the discretion of the clinician. 
In the study by ANASTASATU et al. [14], published in 1969, Eth and Pth were administered in two groups of 26 patients respectively, on top of Cs and viomycin. Gastric intolerance was reported in $46 \%$ of those receiving Eth (three patients interrupted the treatment) and in $23 \%$ only of those receiving Pth (one case interrupted the treatment). Culture conversion occurred in $45 \%$ of those treated with Eth and $70 \%$ of those treated with Pth, respectively. However, the small number of cases did not allow assessment of a statistically significant difference. The authors concluded that the results with Pth could be attributed to its better tolerability.

In another double-blinded study published in 1970 by VerBist et al. [15], $1 \mathrm{~g}$ Pth administered in two daily doses was better tolerated than Eth ( $1 \mathrm{~g}$, two daily doses), although it was associated with more frequent liver toxicity. 130 pulmonary tuberculosis patients were recruited and were prescribed a backbone regimen including Inh and Sm, together with either Pth, Eth, Eth hydrochloride or thiocarlide. The tolerability profile after 7 weeks was poorer in the two groups receiving Eth $(p<0.025)$, although those exposed to Pth showed more biochemical disorders $(\mathrm{p}<0.001)$, especially increased serum transaminase values. Treatment discontinuation was reported in 12 out of 30 patients receiving Pth, in 10 out of 24 patients receiving Eth and 11 out of 25 patients receiving Eth hydrochloride.

Several limitations of this systematic review can be raised. The adoption of the single engine PubMed could have slightly reduced the search sensitivity, although the old and low number of the articles counterbalance this methodological choice. Furthermore, standardised definitions of efficacy, safety and tolerability were not adopted, the selected studies being very old and not following an internationally agreed-upon methodology. The quality of the scientific evidence focused on the clinical comparison between the two drugs was poor according to the grading system we adopted.

In conclusion, although the evidence is limited and rather old, Pth appears to be better tolerated (especially in terms of reduced frequency of gastric adverse events, although Pth-related liver toxicity was reported). According to the publications described here, the efficacy of the two thionamides is similar between studies, although some of them report a higher efficacy of Pth.

The findings of this systematic review seem to suggest, in absence of new evidence, to slightly prefer Pth to Eth in designing MDR-TB regimens. However, the quality of the retrieved scientific evidence is extremely poor, due to the design, implementation, and reporting of the studies dealing with the comparison of the two drugs; consequently, any firm conclusions in terms of preferences should be currently avoided.

@ERSpublications

Prothionamide is slightly better than ethionamide in treating MDR-TB patients http://ow.ly/bSwY300i061

Anna Scardigli ${ }^{1,2,7}$, Jose A. Caminero ${ }^{1,3,7}$, Giovanni Sotgiu ${ }^{4,7}$, Rosella Centis ${ }^{5}$, Lia D'Ambrosio ${ }^{5,6}$ and Giovanni Battista Migliori ${ }^{5}$

${ }^{1}$ MDR-TB Unit, Tuberculosis Division, International Union against Tuberculosis and Lung Disease, Paris, France. ${ }^{2}$ The Global Fund to Fight AIDS, Tuberculosis and Malaria, Geneva, Switzerland. ${ }^{3}$ Pneumology Dept, General Hospital of Gran Canaria "Dr. Negrin", Las Palmas, Spain. ${ }^{4}$ Clinical Epidemiology and Medical Statistics Unit, Dept of Biomedical Sciences, University of Sassari, Medical Education and Professional Development Unit, Sassari, Italy. ${ }^{5}$ WHO Collaborating Centre for TB and Lung Diseases, Fondazione S. Maugeri, Tradate, Italy. ${ }^{6}$ Public Health Consulting Group, Lugano, Switzerland. ${ }^{7}$ These authors contributed equally.

Correspondence: Giovanni Battista Migliori, World Health Organization Collaborating Centre for Tuberculosis and Lung Diseases, Fondazione S. Maugeri, Care and Research Institute, Via Roncaccio 16, 21049, Tradate, Italy.

E-mail: giovannibattista.migliori@fsm.it

Received: March 012016 | Accepted after revision: April 192016 | First published online: June 102016

Conflict of interest: None declared.

Acknowledgments: The authors alone are responsible for the views expressed in this publication and they do not necessarily represent the decisions and policies of their institutions.

\section{References}

1 Falzon D, Jaramillo E, Schünemann HJ, et al. WHO guidelines for the programmatic management of drug-resistant tuberculosis: 2011 update. Eur Respir J 2011; 38: 516-528.

2 World Health Organization. Companion handbook to the WHO guidelines for the programmatic management of drug-resistant tuberculosis. WHO/HTM/TB/2014.11. Geneva, World Health Organization, 2014.

3 Caminero JA, Van Deun A, Fujiwara PI, et al. Guidelines for clinical and operational managment of drug-resistant tuberculosis. Paris, International Union Against Tuberculosis and Lung Disease, 2013.

4 Caminero JA, Scardigli A. Classification of anti-TB drugs: a new potential proposal based on the most recent evidence. Eur Respir J 2015; 46: 887-893.

5 Ahuja SD, Ashkin D, Avendano M, et al. Multidrug resistant pulmonary tuberculosis treatment regimens and patient outcomes: an individual patient data meta-analysis of 9,153 patients. PLoS Med 2012; 9: e1001300. 
Falzon D, Gandhi N, Migliori GB, et al. Resistance to fluoroquinolones and second-line injectable drugs: impact on multidrug-resistant TB outcomes. Eur Respir J 2013; 42: 156-168.

7 Smith C, Abubakar I, Thomas HL, et al. Incidence and risk factors for drug intolerance and association with incomplete treatment of tuberculosis: analysis of national case registers for England, Wales and Northern Ireland, 2001-2010. Thorax 2014; 69: 956-958.

8 Chan ED, Laurel V, Strand MJ, et al. Treatment and outcome analysis of 205 patients with multidrug-resistant tuberculosis. Am J Respir Crit Care Med 2004; 169: 1103-1109.

9 Nathanson E, Gupta R, Huamani P, et al. Adverse events in the treatment of multidrug-resistant tuberculosis: results from the DOTS-Plus initiative. Int J Tuberc Lung Dis 2004; 8: 1382-1384.

10 Chambatte CKI, Haguenauer G, Page G, et al. Essais cliniques duthionamide de lácide alpha-propyl-isonicotinique (1321 TH) dans le traitement de la tuberculose humaine. Tolerence, toxicite viscerale comparees a celles du $1314 \mathrm{TH}$ (a propos de 21 cas traites pendant deux mois et de 70 cas traites pendant trois mois). Rev Tuberc Pneumol 1965; $29: 33$.

11 Cooperative Study Unit on Chemotherapy of Tuberculosis of the National Sanatoria in Japan. Comparison of the clinical usefulness of ethionamide and prothionamide in initial treatment of tuberculosis: tenth series of controlled trials. Tubercle 1968; 49: 281-290.

12 Research Committee of the British Tuberculosis Association. A comparison of the toxicity of prothionamide and ethionamide. Tubercle 1968; 49: 125-134.

13 Fox W, Robinson DK, Tall R, et al. A study of acute intolerance to ethionamide, including a comparison with prothionamide, and the influence of a vitamin B-complex additive in prophylaxis. Tubercle 1969; 50: 125-143.

14 Anastasatu C, Ulpian C, Weiss F. 20th Conference of IUATLD. Comparaison du prothionamide (1321 TH) et de l'éthionamide (1314 TH) dans le traitement de la tuberculose à bacilles résistants. Bull Int Union Tuberc 1970; 43: 14.

15 Verbist L, Cosemans J, Prignot J, et al. 20th Conference of IUATLD. Double blind study on the tolerance to prothionamide and ethionamide in original treatment of tuberculous patients. Bull Int Union Tuberc 1970; 43: 97-108.

Eur Respir J 2016; 48: 946-952 | DOI: 10.1183/13993003.00438-2016 | Copyright (cERS 2016 